\title{
Large-Scale Multidisciplinary Optimization of an Electric Aircraft for On-Demand Mobility
}

John Hwang

NASA Glenn Research Center

Andrew Ning

Brigham Young University, aning@byu.edu

Follow this and additional works at: https://scholarsarchive.byu.edu/facpub

Part of the Mechanical Engineering Commons

\section{Original Publication Citation}

Hwang, J. T., and Ning, A., "Large-Scale Multidisciplinary Optimization of an Electric Aircraft for On-Demand Mobility," AIAA Structures, Structural Dynamics, and Materials Conference, Kissimmee, FL, Jan. 2018. doi:10.2514/6.2018-1384

\section{BYU ScholarsArchive Citation}

Hwang, John and Ning, Andrew, "Large-Scale Multidisciplinary Optimization of an Electric Aircraft for OnDemand Mobility" (2018). Faculty Publications. 2055.

https://scholarsarchive.byu.edu/facpub/2055 


\title{
Large-scale multidisciplinary optimization of an electric aircraft for on-demand mobility
}

\author{
John T. Hwang * \\ Peerless Technologies Corporation, 2300 National Rd, Beavercreek, OH 45324 \\ Andrew Ning ${ }^{\dagger}$ \\ Brigham Young University, Provo, UT 84602
}

\begin{abstract}
Distributed electric propulsion is a key enabling technology for on-demand electric aircraft concepts. NASA's X-57 Maxwell X-plane is a demonstrator for this technology, and it features a row of high-lift propellers distributed along the leading edge of its wing to enable better aerodynamic efficiency at cruise and improved ride quality in addition to less noise and emissions. This study applies adjointbased multidisciplinary design optimization to this highly coupled design problem. The propulsion, aerodynamics, and structures are modeled using blade element momentum theory, the vortex lattice method, and finite element analysis, respectively, and the full mission profile is discretized and analyzed. The design variables in the optimization problem include the altitude profile, the velocity profile, battery weight, propeller diameters, propeller rotational speeds, blade profile parameters, wing thickness distribution, and angle of attack. Optimizations take on the order of $\mathbf{1 0}$ hours, and a $12 \%$ increase in range is observed.
\end{abstract}

\section{Introduction}

On-demand mobility (ODM) for aviation has potential to be a disruptive innovation for the transportation industry. ODM is a term that NASA is using to refer to non-scheduled air transportation for people, goods, and services using small aircraft that leverage several convergent technologies, including electric propulson [1]. ODM would provide significant economic and quality-of-life benefits to the United States by easing congestion in urban areas and providing fast air travel for low-demand routes.

Two specific applications being targeted for ODM are thin-haul commuters and intra-city air taxis. Thin haul commuters would fly routes between smaller cities for which the demand does not justify operations for traditional airlines. Thin-haul is so-named because of the long and thin portion of the demand-versus-distance curve. Thinhaul routes are shorter and of lower demand than the short- and long-haul routes of traditional airlines, which are gradually consolidating towards longer routes and larger aircraft [2]. Existing thin-haul airlines are profitable only with federal funding via the Essential Air Service (EAS) program to aid underserved communities. The envisioned thin-haul commuters would carry up to about 20 passengers on routes of up to $500 \mathrm{~nm}$ [2], and advanced technologies would enable them to be profitable and scale up to a much larger network of routes.

Intra-city air taxis would enable on-demand point-to-point transport within a city or provide daily commuters a faster alternative to driving in highly congested urban areas. These aircraft would carry up to 6 passengers on routes of up to $100 \mathrm{~nm}$ [2]. Since they would serve densely populated areas, intra-city air taxis have more transformative potential, but they require vertical takeoff and landing (VTOL) operation to provide adequate time savings. The technological complexity and requirements for additional infrastructure [3, 4], e.g., helipads on skyscrapers, make thin-haul commuters a more likely platform for the initial deployment of technologies such as electric propulsion.

There are several convergent technologies whose recent advancements enable ODM, but two of the most important are autonomy and electric propulsion. At least partial autonomy is necessary for the long-term scalability of ODM to deal with the crowded airspaces that would result in a full-scale deployment of ODM. Full autonomy would also eliminate the cost and weight of the pilot, which would be significant given the small size of intra-city ODM aircraft.

Electric propulsion has several direct and indirect benefits for both thin-haul commuters and intra-city ODM. First, the conversion of electrical to mechanical energy in an electrical propulsion system has an overall efficiency of more than $95 \%$, while reciprocating and turbine engines have thermal efficiencies of $20-30 \%$. This efficiency advantage translates to lower energy costs and lower environmental impact. Second, electric propulsion is more

\footnotetext{
*Research Engineer, NASA Glenn Research Center, AIAA Member. (hwangjt@umich.edu)

${ }^{\dagger}$ Assistant Professor, Department of Mechanical Engineering, AIAA Senior Member.
} 
amenable to distributed propulsion because power can be easily transmitted via wires, and motors are scale-invariantthey maintain efficiency as they scale down in size, unlike internal combustion engines (ICEs). Distributed electric propulsion (DEP) improves aerodynamics, because high-lift propulsors distributed along the leading edge of the wing increase the effective dynamic pressure, enabling stall-speed requirements to be met with much smaller wings that have lower drag during cruise. The resulting high-aspect-ratio wings also have better aerodynamic efficiency, and the high wing loading reduces sensitivity to gust loads and turbulence. Third, electric motors are more compact and made of fewer parts than ICEs, which makes them simpler, lighter, more reliable, and cheaper to maintain. Electric motors also have a wider operating envelope in terms of efficiency and torque versus RPM, which means that variable-pitch propellers are not required. Fourth, power-to-weight ratio is also several factors better than ICEs, and there is no risk of power lapse depending on atmospheric conditions. In summary, the advantages of electric propulsion include lower costs, lower environmental impact, improved aerodynamics, better ride quality, and more reliability.

With current battery technology, the critical limitation for pure electric propulsion is that batteries have energy densities that are 50-100 times lower than those of jet fuel. Even after factoring in the three-fold engine efficiency advantage for electric propulsion and other first-order effects, there is still 10-20 times less energy available [5], which translates to a smaller range. Other disadvantages of batteries include the high manufacturing cost, the additional infrastructure required, and certification.

There are also significant operational challenges that must be overcome for not just electric propulsion, but ODM in general. These include airspace integration, air traffic interaction, ground infrastructure, community acceptance of noise, and regulations [6]. Developing solutions for the operational challenges continues to be an active topic of research.

Given the promise of electric aircraft, the motivation for this paper is to explore whether modern design optimization methods can provide another source of performance improvement to offset the battery technology and operational challenges. We present a system-level multidisciplinary design optimization study for a conventional takeoff and landing (CTOL) aircraft designed for thin-haul commuter applications. We use NASA's X-57 Maxwell X-plane as the baseline given the availability of data for this concept.

This study improves upon the detail level and accuracy of previous system-level vehicle studies $[7,8,5,9]$ in two ways. First, we consider all relevant disciplines (propeller analysis, wing aerodynamics, structures, energy storage, and mission) using physics-based models, selecting an appropriate level of fidelity to capture first-order effects and tradeoffs. Second, we take an adjoint-based optimization approach, allowing us to use optimization as a design tool without major restrictions in terms of number of disciplines or design variables. The objective is to evaluate whether large-scale multidisciplinary design optimization can be a useful method in the design of electric aircraft.

\section{Approach}

The object of study is NASA's X-57 Maxwell research aircraft, which is shown in Fig. 1. The X-57 is a CTOL electric aircraft with tip-mounted cruise propellors and high-lift propellers distributed along the wing leading edge for takeoff and approach. The X-57 is a retrofit of the Tecnam P2006T developed for the sole purpose of providing flight test data for the DEP technology. Therefore, it is not designed for a traditional metric such as maximum range or endurance; however, we believe it presents a good choice for a first application of large-scale MDO because of the availability of published descriptions and validation data.

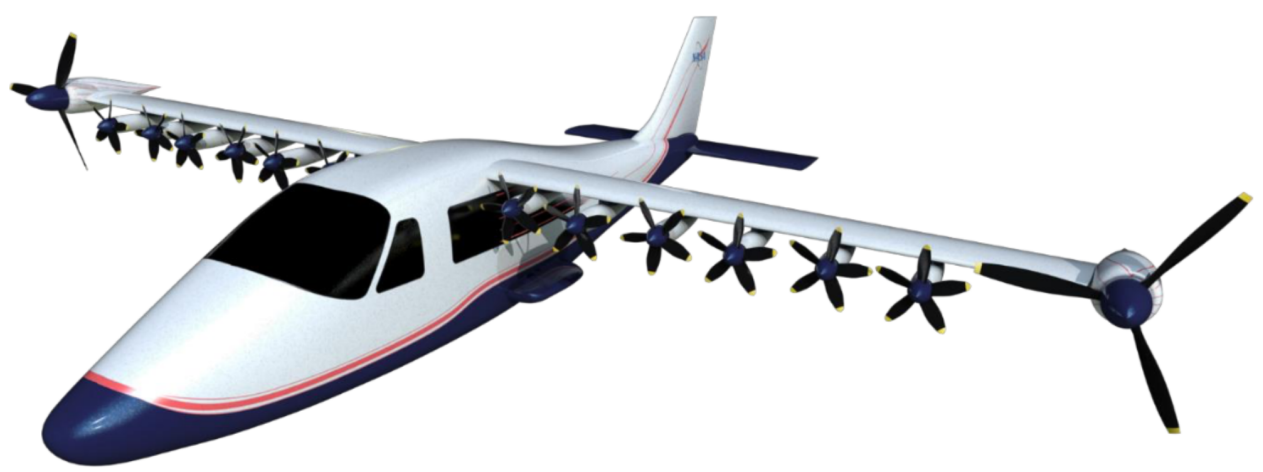

Figure 1: NASA's X-57 Maxwell demonstrator [9]. 
In applying MDO to the X-57, we aim to determine the aspects of the X-57's design and operation to which its performance is the most sensitive. These results would shed light on the potential usefulness of large-scale MDO as a design tool for electric aircraft, given its current capabilities. Such a study would also provide a demonstration of large-scale MDO on a complex aircraft design problem and motivate the development of new methods to handle the computational challenges that arise.

\section{A. Modeling approach}

We pay special attention to the DEP aspect of the X-57, especially the cruise and high-lift propeller designs. With a fixed number of high-lift propellers, changing the propeller diameters changes the wing span since they are designed to precisely cover the entire wing with blown air, i.e., the propellers are positioned to almost overlap. As a result, aerodynamics, structures, and battery models are necessary to capture the dominant tradeoffs. Moreover, since the wing aspect ratio is constrained by the high-lift condition but dominates the cruise performance, it is necessary to at least consider multiple operating conditions if not the full mission profile. Therefore, our disciplines of interest are propeller-wing aerodynamics, structures, weights, energy storage, and mission.

The most important aspect of the model is the propeller-wing aerodynamics, and there is extensive literature in this area for the X-57 and similar propeller-wing concepts. Borer et al. [10] use XROTOR, which models the blades as lifting lines and can perform minimum-induced-loss blade design, and they [11] later combine it with blade section data generated by XFOIL, a 2-D panel method coupled to a boundary layer solution. In both studies, they perform design exploration using Latin hypercube sampling, parametrizing the blade profiles with low-order polynomials. These models are later combined with AVL, a vortex lattice method implementation, to predict lift and induced drag [9]. Patterson et al. [12] develop a design method for high-lift propeller design, taking into account the assumption that uniform axial induced velocity profiles are advantageous for lift augmentation.

Many studies have also looked at higher-order or high-fidelity methods. Distributed vorticity element (DVE) methods $[13,14,15]$ use higher-order elements and improve accuracy over the BEMT-VLM approach through wake relaxation while avoiding singularities, which are typical of traditional free-wake methods. These methods also have the advantage of directly modeling both the blade and wing surfaces directly, avoiding the need to model the interactions with approximations. Computational fluid dynamics (CFD) has also been used to model the wing aerodynamics in the wake of the propellers [16].

Here, we use blade element momentum theory (BEMT) for the propeller analysis and the vortex lattice method (VLM) for the wing analysis. The BEMT-VLM combination provides a simple and fast physics-based model that is appropriate for a system-level design study. The CFD and DVE methods offer improvements in accuracy, but their computation time and complexity make them ill-suited for a system-level multidisciplinary optimization study with relatively fast turnaround time.

We perform design optimization considering the full mission profile, with climb, cruise, and descent modeled continuously. The altitude and velocity profiles are parametrized with design variables, and the vertical and horizontal equations of motion are enforced at each point in the mission, following Kao et al. [17] In the context of commercial aircraft design, Hwang and Martins perform simultaneous design and mission optimization using CFD, replacing it with a surrogate model re-trained each optimization iteration to avoid the large number of CFD evaluations [18]. Here, we forego the surrogate model and evaluate the BEMT and VLM models directly at each mission point. Falck et al. [8] performed a similar optimization study for the X-57, but focused on the electric propulsion system and thermodynamics, rather than the airframe.

\section{B. Optimization approach}

For the purposes of discussion, we characterize optimization as large-scale when there are more than $\sim 10$ design variables. For large-scale optimization or MDO, gradient-based optimization algorithms are the most appropriate, because they typically have orders of magnitude lower solution time than gradient-free algorithms with $\mathcal{O}(10)$ or more design variables. Moreover, gradient-based algorithms find solutions that satisfy nonlinear constraints in highdimensional spaces with precision that gradient-free algorithms cannot match. Recent advances greatly simplify the implementation of highly efficient gradient-based MDO algorithms; therefore, we are interested in applying them to the strongly-coupled and complex design problem that electric aircraft present.

A key enabler for large-scale MDO is derivative computation using the adjoint method because it reduces the computational cost of computing derivatives by a factor of $n$, where $n$ is the number of design variables. However, the adjoint method is complex and time-consuming to implement, especially in the presence of multiple disciplines. Moreover, it requires models of a certain structure-the discipline(s) must contain states computed implicitly by solving a system of equations. Alternatively, if there are multiple disciplines that explicitly compute their outputs but 
have feedback loops among each other, another derivative computation method is the most efficient, the GSE2 (global sensitivity equations) [19]; on the other hand, if there are no feedback loops, the chain rule is sufficient.

Recently, these methods were unified through a matrix equation [20] that inherently reduces to the adjoint method, GSE2, chain rule, or other hybrid methods based on the model structure. This matrix equation results from the application of the inverse function theorem to a nonlinear system monolithically representing the multidisciplinary model. Using this representation and unification, a software framework can be built to automate some of the effort of computing derivatives - this approach has been named MAUD (modular analysis and unified derivatives) [21]. With MAUD, the computation of gradients required for optimization proceeds in two stages: (1) the computation of partial derivatives of each component-a modular unit of code in the model — and (2) the assembly of the partial derivatives into total derivatives that capture the interactions between components, i.e., disciplines.

MAUD has been implemented in NASA's OpenMDAO software framework [22], and it has been used for satellite, wind turbine, airline allocation, and engine cycle MDO problems. In this paper, we implement all of our models within OpenMDAO and use it to simplify the development of our electric aircraft model for large-scale MDO. The models for each discipline are natively implemented in OpenMDAO; i.e., we avoid wrapping an external software package, and all partial derivatives are hand-differentiated. While this required significant effort, hand-differentiated partial derivatives yield maximum accuracy and efficiency. Given the components and partial derivatives, OpenMDAO uses MAUD to assemble the total derivatives through the adjoint method, GSE2, chain rule, or a hybrid thereof.

\section{Disciplinary models}

In this section, we describe the models for the propeller, wing aerodynamics and drag buildup, wing structural response, weight buildup, battery performance, and mission analysis. The propeller is modeled using blade element momentum theory, the wing using the vortex lattice method, and the structure using 6-DOF spatial beam elements.

\section{A. Propeller model}

The propeller is modeled using blade element momentum theory (BEMT). BEMT discretizes the propeller radially, providing sufficient modeling fidelity to predict the thrust, torque, lift, and the 3-D induced velocity field given blade airfoil data, chord and twist profiles, inflow velocity vector, and rotational velocity setting. We model the propeller slipstream using analytical solutions assuming an elliptical load distribution on the blade, while considering its deflection due to the angle of the freestream velocity vector. Each of the X-57's propellers is separately modeled, and their aggregate influence on the wing aerodynamics is modeled as an inflow velocity component in the vortex lattice method.

\section{Blade element momentum theory}

Blade element momentum theory (BEMT) combines momentum theory, which uses a control-volume analysis, and blade element theory, which is based on known airfoil data at each section. We equate the results of the two theories at each radial section to compute the axial and tangential induced velocities, $a_{x}$ and $a_{y}$, respectively, which are normalized velocities induced by the propeller in the normal and circumferential directions.

MOMENTUM THEORY In momentum theory, we use conservation laws on a control volume extending far upstream and downstream of the propeller, modeled as a disc. Assuming known induced velocities, momentum theory predicts

$$
\begin{aligned}
& d T=C_{T} \frac{1}{2} \rho V_{x}^{2} d A, \quad \text { with } \quad C_{T}=4 F a_{x}\left(1+a_{x}\right), \\
& d Q=C_{Q} \frac{1}{2} \rho V_{x}^{2} d A r, \quad \text { with } \quad C_{Q}=4 F a_{y}\left(1+a_{x}\right) \frac{V_{y}}{V_{x}},
\end{aligned}
$$

where $d T$ and $d Q$ are the differential thrust and torque, $C_{T}$ and $C_{Q}$ are the thrust and torque coefficients, $V_{x}$ and $V_{y}$ are the normal and circumferential velocity components seen by the propeller, $d A$ is the area for a radial slice of the propeller, $\rho$ is air density, $F$ is the loss factor, and $r$ is the radial position.

BLADE ELEMENT THEORY Blade element theory uses specified airfoil polars to compute the forces on a blade-byblade basis given their local angles of attack at radial sections. The differential thrust and torque predicted at a radial 
section are

$$
\begin{aligned}
& d T=B C_{x} \frac{1}{2} \rho V^{2} c d r \\
& d Q=B C_{y} \frac{1}{2} \rho V^{2} c r d r
\end{aligned}
$$

where $B$ is the number of blades, $c$ is the chord, $d r$ is the differential radial width, and $V$ is the magnitude of the velocity seen by the section. We note that $V$ depends on the induction factors: $V=\sqrt{\left[V_{x}\left(1+a_{x}\right)\right]^{2}+\left[V_{y}\left(1-a_{y}\right)^{2}\right]}$. The coefficients are given by

$$
\left[\begin{array}{l}
C_{x} \\
C_{y}
\end{array}\right]=\left[\begin{array}{cc}
\cos \phi & -\sin \phi \\
\sin \phi & \cos \phi
\end{array}\right]\left[\begin{array}{l}
C_{l} \\
C_{d}
\end{array}\right]
$$

where the local inflow angle $\phi$ is computed from $\phi=\theta-\alpha$, where $\theta$ is the local twist angle.

COUPLED SOLUTION We equate the torque and thrust predicted by momentum theory and blade element theory, and after some manipulation, we obtain

$$
\begin{aligned}
& a_{x}=\frac{C_{x} \sigma}{4 F \sin ^{2} \phi-C_{x} \sigma}, \\
& a_{y}=\frac{C_{y} \sigma}{2 F \sin 2 \phi+C_{y} \sigma},
\end{aligned}
$$

where $\sigma$ is the blade solidity, given by $\sigma=B c /(2 \pi r)$. Since the right-hand sides of the above equations depend on $\phi$ and $V$, which depend on $a_{x}$ and $a_{y}$, this is a coupled system of equations. However, $V$ appears only in the Reynolds number, and is only weakly dependent on the induction factors, so we can approximate this system as a single equation in only $\phi$, which allows us to use a bracketed search method that is provably convergent [23]. The residual function, rearranged to avoid singularities, is given by

$$
V_{y}\left(4 F \sin ^{2} \phi-C_{x} \sigma\right)-V_{x}\left(2 F \sin 2 \phi+C_{y} \sigma\right)=0,
$$

which is valid for $V_{x}>0, V_{y}>0$.

\section{Blade airfoil data}

The X-57 uses the MH-117 and MH-114 for the cruise and high-lift propeller's airfoils, respectively. XFOIL-generated data for these airfoils are obtained from an airfoil database ${ }^{a}$ with angles of attack until and slightly past stall and Reynolds numbers ranging from 50,000 to 1,000,000. To obtain full-range polars, this data is extended post-stall using the Viterna method, which uses an inviscid flat plate assumption. The resulting $C_{l}$ and $C_{d}$ data as a function of $\alpha$ and $R e$ are interpolated using a minimal-energy tensor-product spline surrogate model [24]. As shown in Fig. 2, this surrogate model captures the trends while providing a small, controllable degree of smoothing, which helps for gradient-based optimization.

\section{Slipstream modeling}

The induced velocity field in the propellers' slipstream is modeled using analytical approximations. The slipstream contraction is approximated [25] using

$$
R(x)=R \sqrt{\frac{1+a_{x}}{1+a_{x}\left(1+\frac{x}{\sqrt{R^{2}+x^{2}}}\right)}},
$$

which is derived assuming a uniform axial load distribution. As noted by Veldhuis, there are errors in this approximation due to nacelle effects [25], but we use it as a first-order model that is at least better than neglecting slipstream contraction entirely.

\footnotetext{
${ }^{\mathrm{a}}$ www.airfoiltools.com
} 

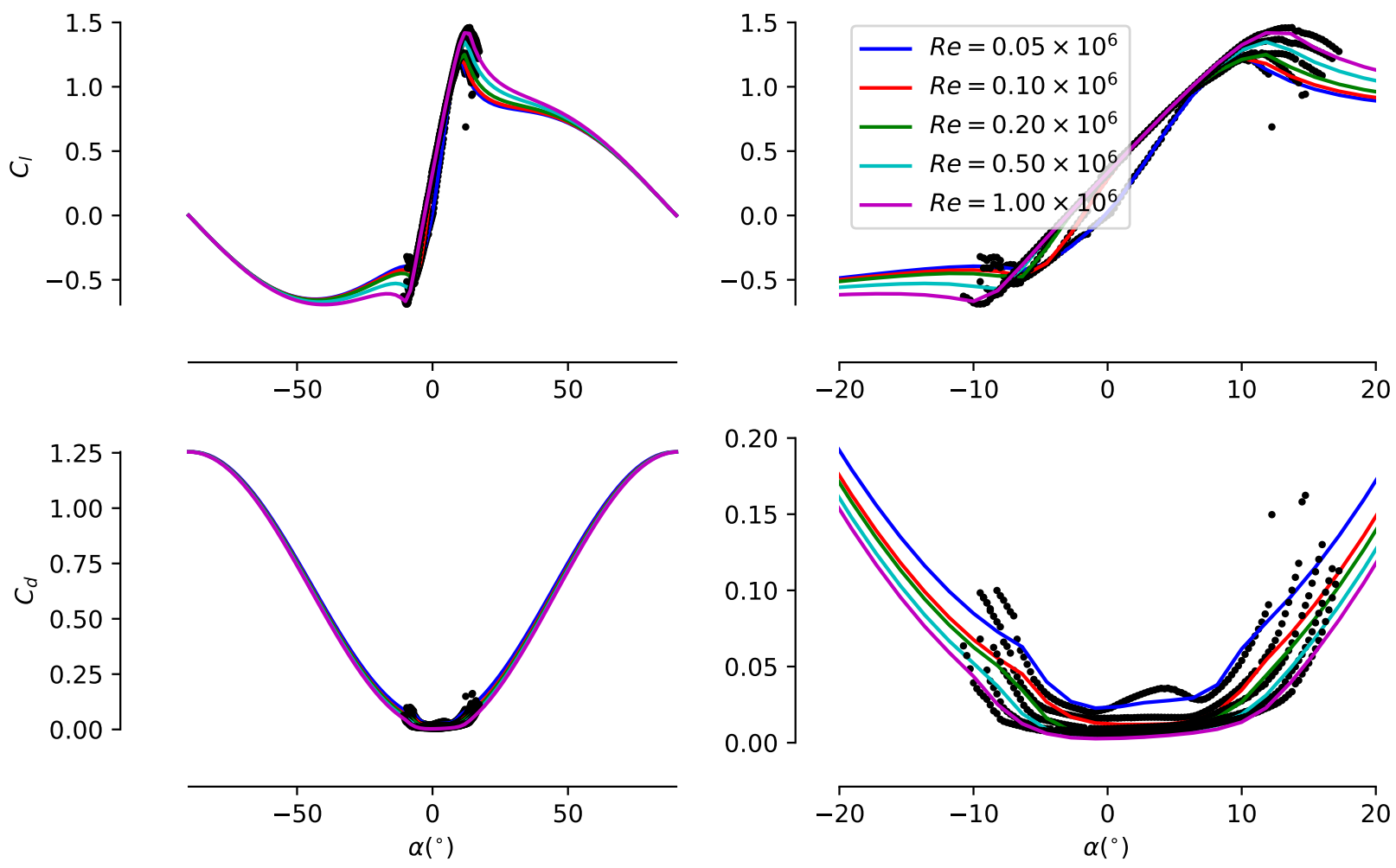

Figure 2: 2-D polars for the X-57's cruise propeller airfoil (MH-117) at various Reynolds numbers. The current approach smoothes the data and extrapolates past stall using an analytical flat-plate model.

For the evolution of the axial and radial components of induced velocity, we use the analytical solution of Conway [26] for elliptically-loaded blades, following Alba [27]. These components are given by

$$
\begin{aligned}
& V_{r}(r, x)=V_{x}(r, 0)\left[\frac{|x| R(x)}{2 r \sqrt{R(x)^{2}-r^{2}}}\left(\frac{1}{a}-a\right)-\frac{r}{2 \sqrt{R(x)^{2}-r^{2}}} \arcsin \left(\frac{2 R(x)}{b}\right)\right], \\
& V_{x}(r, x)=V_{x}(r, 0)\left[2-\frac{a R(x)}{\sqrt{R(x)^{2}-r^{2}}}+\frac{x}{\sqrt{R(x)^{2}-r^{2}}} \arcsin \left(\frac{2 R(x)}{b}\right)\right],
\end{aligned}
$$

where

$$
\begin{aligned}
& a=\sqrt{\frac{\sqrt{\left(R(x)^{2}-x^{2}-r^{2}\right)^{2}+4 R(x)^{2} x^{2}}+\left(R(x)^{2}-x^{2}-r^{2}\right)}{2 R(x)^{2}}}, \\
& b=\sqrt{x^{2}+(R(x)+r)^{2}}+\sqrt{x^{2}+(R(x)-r)^{2}}
\end{aligned}
$$

The value of $V_{x}(r, 0)$ is computed from $V_{x}(r, 0)=V_{x_{0}} \sqrt{R(x)^{2}-r^{2}} / R(x)$, where $V_{x_{0}}$ is the axial induced velocity at the disk, averaged radially. The axial velocity component is considered zero for $r>R(x)$. Likewise, the tangential velocity is also considered zero for $r>R(x)$, and since modeling its evolution in $x$ is more complicated, we use a swirl recovery factor (SRF) of 0.5, following Veldhuis [25]. The SRF is a multiplier on the tangentional induced velocity computed at the disk and it captures the influence of the wing on dampening the swirl in the slipstream.

\section{Verification and model limitations}

We partially verify the BEMT model—namely, its implementation and its hand-derived partial derivatives—by performing optimization with a single blade. The objective function being maximized is the power output, given by $P_{\text {out }}=T V$, and the constraint is a specified value of the power used, given by $P_{r e q}=Q \omega$, where $\omega$ is the angular velocity in radians per second. The chord and twist distributions are parametrized with $10 \mathrm{~B}$-spline control points, and 
the blade is modeled with 25 radial sections. We perform the optimization on the X-57's baseline cruise propellers, which have a 5-ft diameter, 1-ft hub diameter, and an RPM of 2250. The power required is constrained to be $60 \mathrm{~kW}$, which sizes it for climb. The optimized profile is shown in Fig. 3.
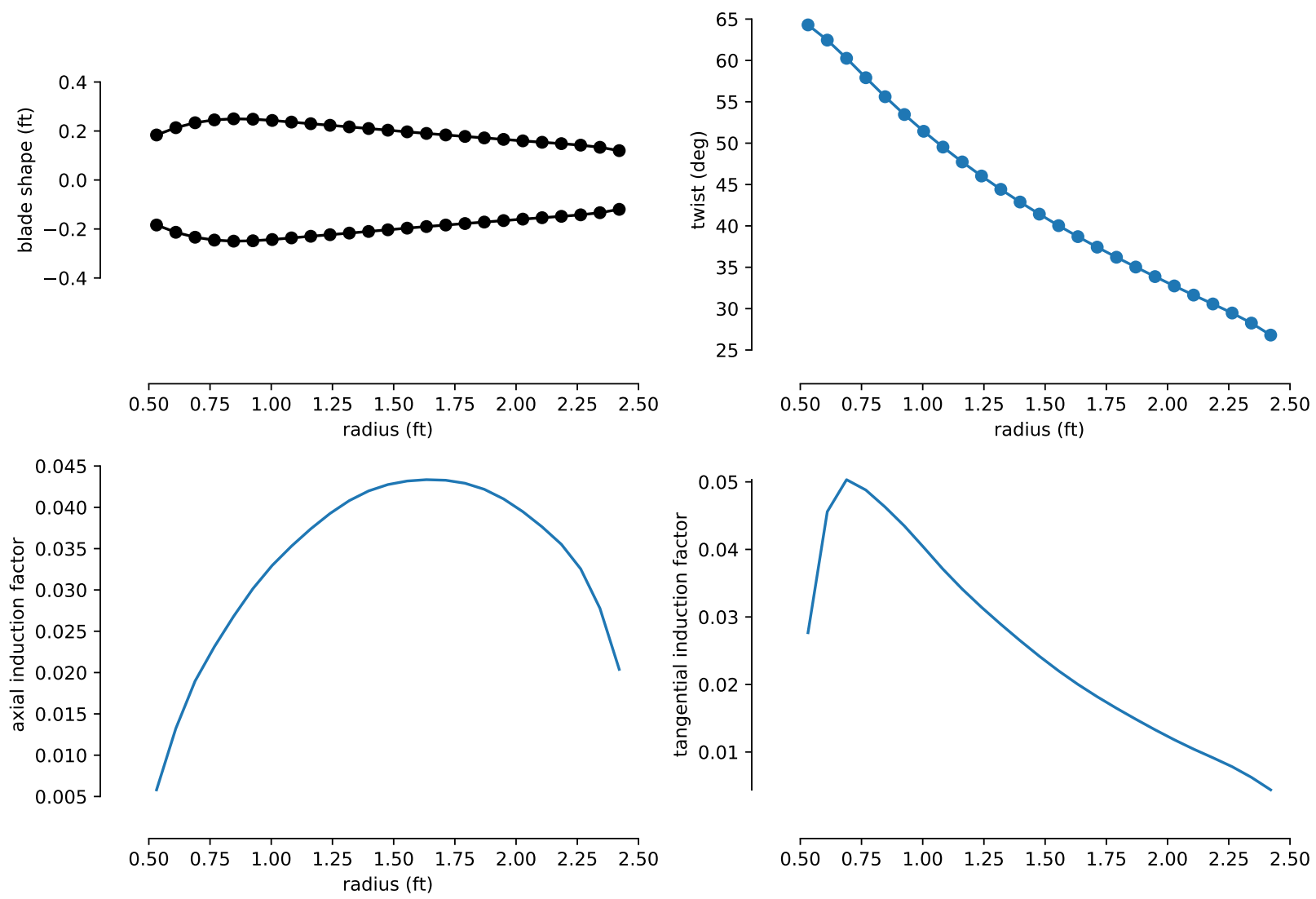

Figure 3: Results of a chord and twist optimization for the X-57's cruise propeller.

The primary limitations of the propeller model are due to: neglecting the effects of the hub, the inviscid assumption for the full-range airfoil polars, the use of the constant swirl recovery factor, and the elliptical loading assumption used for the slipstream model. The hub increases the inflow velocity in the blade analysis and influences the slipstream velocity field, especially the amount of contraction. As a result, we keep the hub diameter fixed in the optimization studies. The inviscid flat-plate approximation for the airfoil polars is a necessary assumption if not resorting to much higher-fidelity models. The slipstream assumptions have room for improvement without significant increase in model complexity and cost; refinement and detailed validation are relevant areas for future work.

\section{B. Aerodynamics model}

The wing lift and induced drag are modeled using the vortex lattice method, adapted to consider the influence of the propeller-induced velocity field. Additional lift and drag terms are added for the lift at zero angle of attack, parasitic drag, and the drag components of the rest of the aircraft.

\section{Vortex lattice method}

The vortex lattice method (VLM) computes lift and induced drag of a set of lifting surfaces by discretizing them spanwise and chord-wise, and modeling them as a superposition of horseshoe vortices. We use the VLM implementation in OpenAerostruct [28], which is an open-source coupled aerostructural optimization tool using VLM and FEA. Here, we use a ring-vortex formulation, where the fore and aft legs of a ring are located at the quarter-chord mark of the relevant panels in the discretization. The aft-most row of rings extends downstream to infinity. 
Each ring has a strength that induces a velocity field, as given by the Biot-Savart law. For each ring, there is a corresponding equation that constrains its value, and this equation is obtained by enforcing flow tangency at the three-quarter-chord point - that the net velocity at this point has no component normal to the panel. This equation is given by

$$
\vec{v} \cdot \hat{n}=\left(\vec{v}_{f s}+\vec{v}_{i}\right) \cdot \hat{n}=0
$$

where $\vec{v}$ is the net velocity, $\bar{v}_{f s}$ is the freestream velocity, $\vec{v}_{r}$ is the velocity components due to the rings, and $\hat{n}$ is the normal vector for the panel. Defining $A I C$ has the aerodynamic influence coefficient matrix and $\Gamma_{r}$ as the vortex strengths of the rings, we can rearrange to obtain the system of linear equations,

$$
A I C \Gamma_{r}=-\vec{v}_{f s} \cdot \hat{n} .
$$

After solving for $\Gamma_{r}$, the Biot-Savart law can again be used to compute the net velocity, this time at the quarter-chord point, and the Kutta-Joukowski equation allows us to compute the forces on the panels:

$$
\vec{F}=\rho \Gamma_{r}\left(\vec{v}_{f s}+\vec{v}_{i}\right) \times \vec{l},
$$

where $\vec{F}$ is the net force from which we can compute lift and drag, $\rho$ is the local air density, and $\vec{l}$ is the bound vortex vector for the panel.

For the high-lift configuration, we assume Fowler flaps with $25 \%$ additional area and $30^{\circ}$ flap deflection. We model this using additional chord-wise panels in the VLM mesh where the aft-most panels represent the flaps. Stall is considered simply by limiting each section's lift coefficient to a chosen $C_{l_{\max }}$ value. We use a KreisselmeierSteinhauser function to smoothly approximate the maximum function.

\section{Slipstream influence}

We now describe how the VLM equations are modified to consider the propeller-induced velocities. The axial, tangential, and radial components in the slipstream are first transformed into the aircraft body-fixed frame. Let us denote the resulting induced velocities as $\vec{v}_{p}$. The flow tangency condition is modified by simply augmenting this additional velocity term as follows:

$$
\vec{v} \cdot \hat{n}=\left(\vec{v}_{f s}+\vec{v}_{i}+\vec{v}_{p}\right) \cdot \hat{n}=0 .
$$

However, for the Kutta-Joukowski equation, we only augment the component of $\vec{v}_{p}$ that is normal to the freestream, following Alba [27]. Therefore, the modified equation is

$$
\vec{F}=\rho \Gamma_{r}\left(\vec{v}_{f s}+\vec{v}_{i}+\vec{v}_{p}-\vec{v}_{p} \cdot \vec{v}_{f s}\right) \times \vec{l} .
$$

\section{Aerodynamic coefficients and validation}

The VLM analysis only captures lift at non-zero angle of attack and induced drag. Camber is added to the VLM mesh to obtain the expected lift coefficient at zero angle of attack. This is determined based on 2-D lift coefficient of the NASA GA(W)-2 airfoil at $2^{\circ}$ angle of incidence as predicted by XFOIL, which is 0.74 , and the value predicted using CFD in the literature [29], which is roughly 0.8 .

Based on the 2-D drag coefficient data from XFOIL, we use a constant parasitic wing drag estimate of $C_{D_{p}}=0.01$. The parasitic drag is relatively constant for small angles of attack, and at larger angles of attack, the error is reduced because the induced drag dominates. The parasitic drag coefficient is adjusted based on using the blown velocity with the axial contributions from the propellers' slipstreams rather than the freestream velocity for the normalization. Another constant term of $C_{D_{0}}=0.0250$ is added to the drag coefficient to account for the contributions from the fuselage, horizontal and vertical stabilizers, and landing gear.

We validate the aerodynamics model (minus the $C_{D_{0}}$ term) against RANS CFD predictions [29, 30] using OVERFLOW, STAR-CCM+, and FUN3D in Fig. 4. As stated previously, the camber is adjusted to match the lift coefficient at zero angle of attack. For the cruise configuration with no high-lift nacelles, we find that the lift curve slope matches well and the drag polar falls in between the predictions with fully turbulent and transition models [30]. For the high-lift configuration, we include the propeller force components, and we find that the lift curve slope is under-predicted at low angles of attack. However, for both the lift and drag, the values fall within the ranges of variation of the CFD predictions at high angles of attack, which is where the model is relevant. Our conclusion is that the aerodynamic model shows good agreement overall and that it provides sufficient accuracy for a system-level optimization study. 

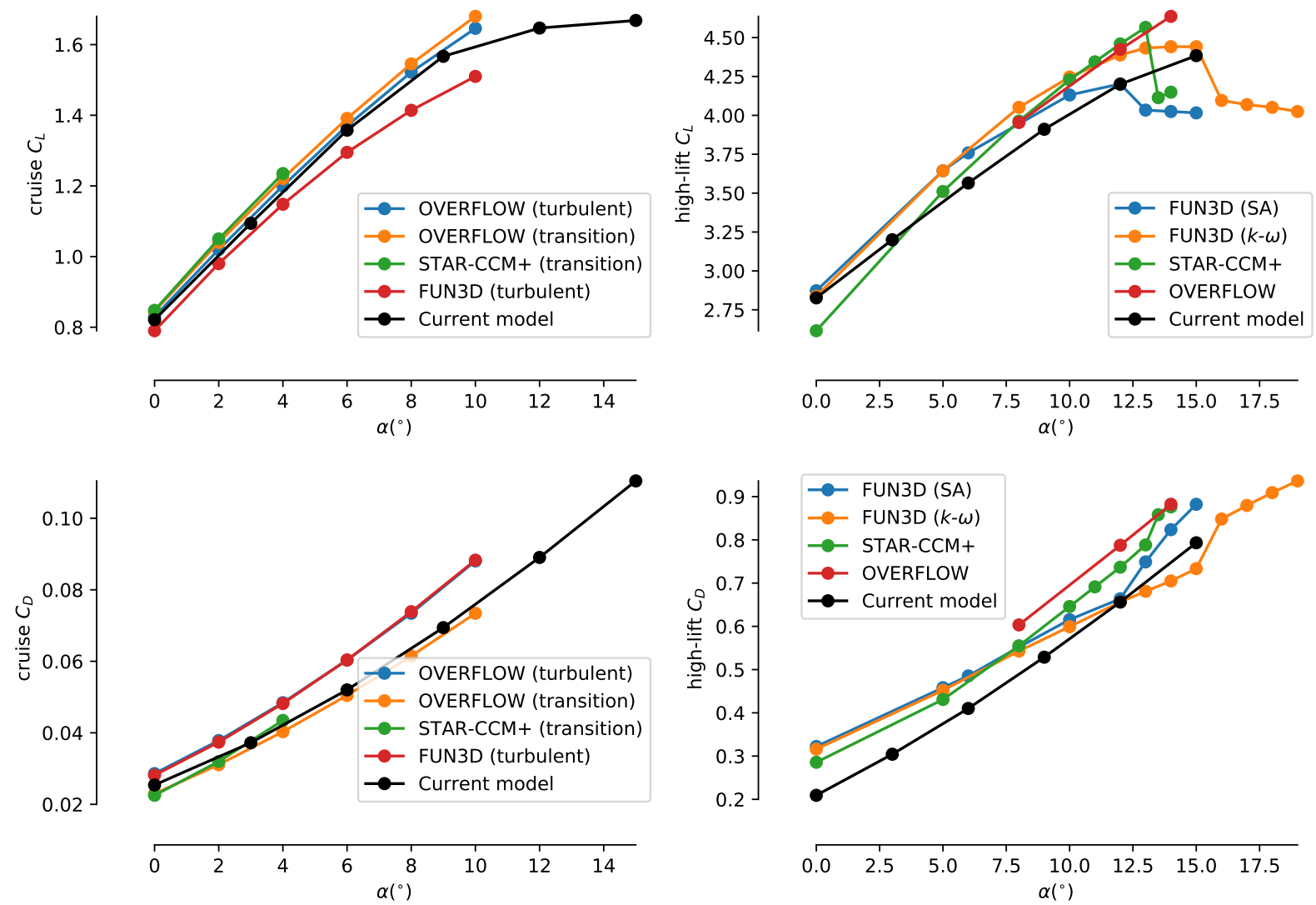

Figure 4: The propeller-wing aerodynamics model shows good agreement with CFD data except for the drag at high angles of attack. The cruise data is from Borer et al. [29] and the high-lift data is from Deere et al. [30].

\section{Structures and weights}

The structural analysis is important because of the effects of changing the wing span on the weight of the airframe. We model the wing structure using 6-DOF spatial beam elements that support axial, bending, and torsional loads. The same span-wise discretization is used as in the VLM mesh to facilitate load transfer from the VLM to the finite element analysis. For simplicity, we assume the elements have a tubular cross-section, and the thickness distribution is parametrized with design variables in the optimization problem. Von Mises stress constraints are enforced with Kreisselmeier-Steinhauser constraint aggregation to reduce the computational cost [31]. Like the VLM model, the FEA model is part of the OpenAerostruct package [28].

For the weights model, we assume a fixed value of $7300 \mathrm{~N}$ for the empty weight not including the battery weight and the weight of the wing's primary structure. The latter is computed from the thickness distribution given by the design variables.

\section{Mission analysis}

The mission profile is discretized, and the horizontal and vertical equations of motions are enforced at every point. These equations are

$$
\begin{aligned}
& T \cos \alpha-D-W \sin \gamma-m \dot{v}_{y} \sin \gamma-m \dot{v}_{x} \cos \gamma=0, \\
& L+T \sin \alpha+m \dot{v}_{x} \sin \gamma-W \cos \gamma-m \dot{v}_{y} \cos \gamma=0,
\end{aligned}
$$

where $T, L, D$, and $W$ are the total aircraft thrust, lift, drag, and weight, respectively, $\alpha$ is the angle of attack, $\gamma$ is the climb angle, $m$ is the total aircraft mass, and $\dot{v}_{x}$ and $\dot{v}_{y}$ are the horizontal and vertical components of acceleration in the Earth-fixed frame. The equations of motion are aligned with the flow direction. The MDO results considering the full mission profile discretize the mission with 10 points. 


\section{Baseline description}

For the results in this paper, we use Rev. 3.3 of the X-57 as the baseline design [9]. Table 1 lists the overall aircraft parameters and dimensions used for the tip-over constraint, which ensures that there is sufficient clearance between the cruise propellers and the ground. Table 2 lists the parameters for the baseline wing. The NASA GA(W)-2 has a thickness-to-chord ratio of $15 \%$, but we assume a modified version of this airfoil that has a thickness-to-chord ratio of $13 \%$, following Borer. Table 3 lists the parameters for the cruise and high-lift propellers. All 12 high-lift propellers are assumed to be identical in design, even during optimization. We note that these tables provide baseline values only; for some of the parameters, the values are allowed to vary during optimization, either directly because they are design variables or as a function of other design variables.

As stated in Tab. 1, we assume a battery energy density of $130 \mathrm{Whr} / \mathrm{kg}$ and a battery power density of $500 \mathrm{~W} / \mathrm{kg}$. We choose these numbers based on current battery technology rather than predicting the capabilities of batteries in the future, as is done in many electric aircraft studies. However, we do not consider the losses due to the imperfect efficiency of the electrical propulsion system, i.e., the motors, and we do not consider reserve energy requirements for missions. Therefore, we can interpret the values in Tab. 1 as effective battery energy and power densities given today's battery technology.

\begin{tabular}{lll}
\hline Property & Value & SI units \\
\hline Cruise speed & $150 \mathrm{knots}$ & $77.2 \mathrm{~m} / \mathrm{s}$ \\
Cruise altitude & $8000 \mathrm{ft}$ & $2440 \mathrm{~m}$ \\
Gross weight & $3000 \mathrm{lb}$ & $13300 \mathrm{~N}$ \\
Battery energy density & $130 \mathrm{Whr} / \mathrm{kg}$ & $468 \mathrm{~kJ} / \mathrm{kg}$ \\
Battery power density & $500 \mathrm{~W} / \mathrm{kg}$ & \\
\hline
\end{tabular}

\begin{tabular}{lll}
\hline Property & Value & SI units \\
\hline Fuselage width & $4 \mathrm{ft}$ & $1.2 \mathrm{~m}$ \\
Main landing gear width & $7 \mathrm{ft}$ & $2.1 \mathrm{~m}$ \\
Wing height & $7 \mathrm{ft}$ & $2.1 \mathrm{~m}$ \\
Tip-over constraint & $20^{\circ}$ &
\end{tabular}

Table 1: Overall aircraft parameters for the Rev 3.3 X-57. The properties on the right are used for enforcing the tip-over constraint.

\begin{tabular}{lll}
\hline Property & Value & SI units \\
\hline Span & $31.6 \mathrm{ft}$ & $9.6 \mathrm{~m}$ \\
Area & $66.7 \mathrm{ft}^{2}$ & $6.2 \mathrm{~m}^{2}$ \\
Aspect ratio & 15 & \\
Airfoil & NASA GA(W)-2 & \\
\hline
\end{tabular}

\begin{tabular}{lll}
\hline Property & Value & SI units \\
\hline Root chord & $2.48 \mathrm{ft}$ & $0.76 \mathrm{~m}$ \\
Tip chord & $1.74 \mathrm{ft}$ & $0.53 \mathrm{~m}$ \\
Reference chord & $2.13 \mathrm{ft}$ & $0.65 \mathrm{~m}$ \\
Thickness-to-chord & 0.15 & \\
\hline
\end{tabular}

Table 2: Wing parameters for the Rev 3.3 X-57.

\begin{tabular}{lll}
\hline Property & Value & SI units \\
\hline Diameter & $5 \mathrm{ft}$ & $1.5 \mathrm{~m}$ \\
Hub diameter & $1 \mathrm{ft}$ & $0.3 \mathrm{~m}$ \\
Cruise RPM & $2250 \mathrm{RPM}$ & $236 \mathrm{rad} / \mathrm{s}$ \\
Airfoil & MH117 & \\
Number of blades & 3 & \\
\hline
\end{tabular}

Cruise propellers

\begin{tabular}{lll}
\hline Property & Value & SI units \\
\hline Diameter & $1.8 \mathrm{ft}$ & $0.55 \mathrm{~m}$ \\
Hub diameter & $0.472 \mathrm{ft}$ & $0.14 \mathrm{~m}$ \\
Max RPM & $4760 \mathrm{RPM}$ & $498 \mathrm{rad} / \mathrm{s}$ \\
Airfoil & MH114 & \\
Number of blades & 5 & \\
\hline
\end{tabular}

High-lift propellers

Table 3: Properties for the cruise and high-lift propellers.

\section{Optimization results}

In this section, we present the optimization formulation and results. We use two formulations: multi-point (MP) optimization and full-mission (FM) optimization. We first describe the two formulations, and then present the MP and FM results separately. 


\section{A. Optimization problem formulation}

We solve two types of optimization problems: propulsive-aerodynamic-structural optimization at the cruise condition with a high-lift sizing condition and propulsive-aerodynamic-structural-mission optimization considering a full mission profile. We refer to the first type as multi-point (MP) optimization and to the second type as full-mission (FM) optimization. The FM formulation discretizes the mission profile with 10 points. For both the MP and FM formulations, we solve a series of four problems (referred to as case $0,1,2,3$ ) that successively add more design variables. The optimization problem statements for all problems are given in Tab. 4.

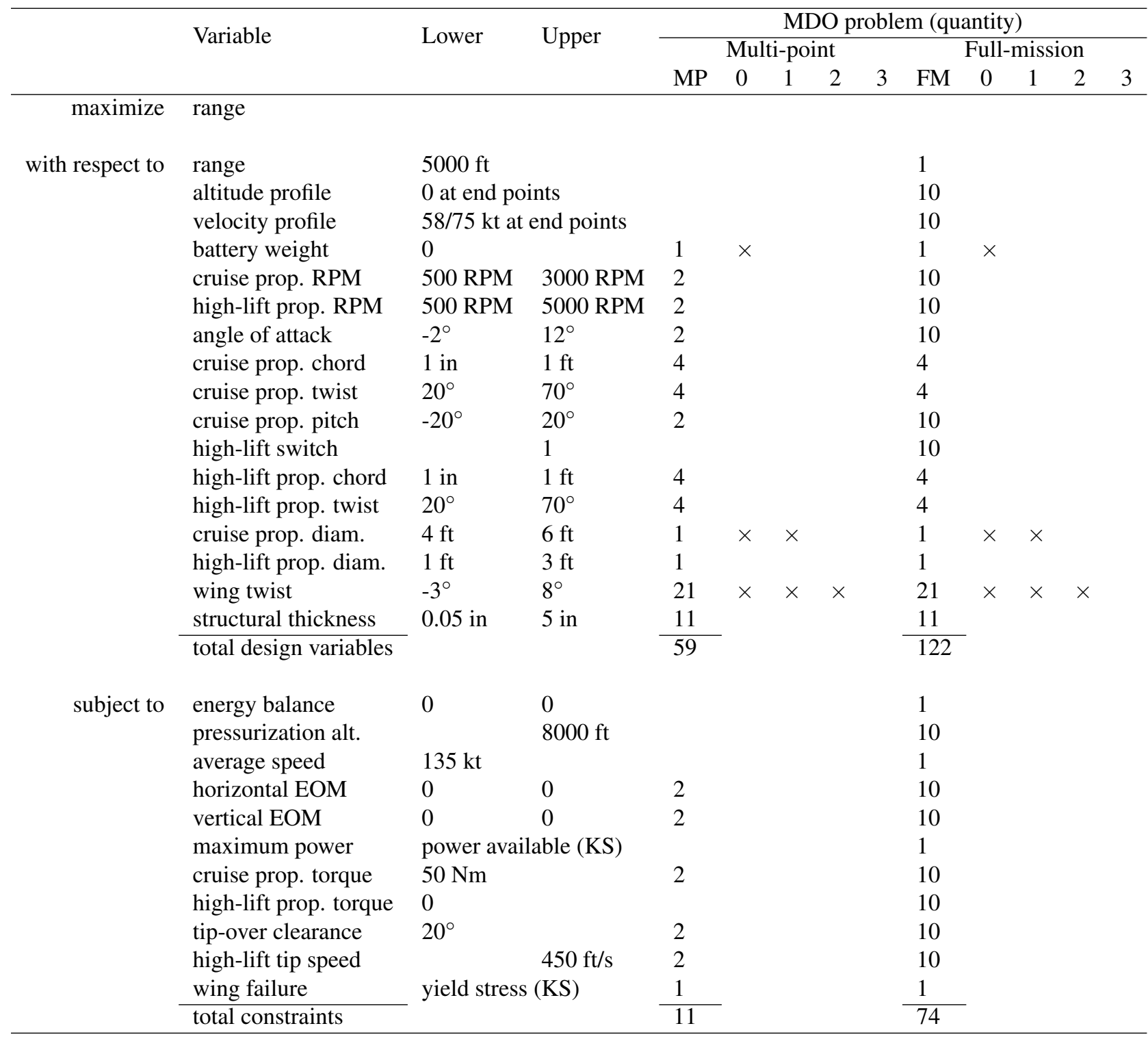

Table 4: MDO problem statements.

For both formulations, there are four sub-problems. The first sub-problem (i.e., MP0 and FM0) is a baseline optimization where the only included design variables are the ones necessary to achieve a feasible point that satisfies the constraints. The range value from this optimization provides a reference for comparison for the other three subproblems. The second sub-problem (i.e., MP1 and FM1) adds battery weight as a design variable. The third subproblem (i.e., MP2 and FM2) adds cruise propeller diameter as a design variable in addition to battery weight. The fourth sub-problem (i.e., MP3 and FM3) includes wing twist distribution in addition to battery weight and cruise propeller diameter. 
In all problems, the objective function being maximized is range. In the multi-point problems, range is computed from the power consumption and velocity at the cruise point, while in the full-mission problems, range is a design variable determined by the constraint that the energy consumption over the mission profile is equal to the energy available from the battery.

We now discuss the design variables and constraints that are common to the MP and FM formulations. The battery weight design variable allows the optimizer to find a compromise that trades off energy and aircraft weight. The cruise and high-lift propeller RPMs are allowed to vary independent at each mission point (just the two points in the MP case) to provide enough freedom to satisfy the horizontal equation of motion while determining the optimal amount of lift augmentation. Both are assigned a lower bound of 500 RPM to avoid the significant degradation of motor efficiency that would occur at lower rotational speeds. Both are also assigned upper bounds because of the limitations of the respective motors. The angle of attack design variable allows the optimizer to satisfy the vertical equation of motion at all mission points. The cruise and high-lift propellers' chord and twist distributions are included in the optimization problem to allow them to be sized simultaneously while the other variables change in the MDO problem. They are parametrized with 4 control points of a 4 th order B-spline curve. The cruise propeller is variable-pitch, so another design variable vector is the pitch angle at all mission points. The cruise and high-lift propeller diameters are permitted to vary roughly $1 \mathrm{ft}$ from their baseline values of $5 \mathrm{ft}$ and $1.8 \mathrm{ft}$, respectively. The wing twist and structural thickness variables round out the set of design variables. These parametrize the 101 span-wise panels and the 100 beam elements with 21 and $11 \mathrm{~B}$-spline control points, respectively.

In terms of constraints, the horizontal and vertical equations of motion are enforced at all mission points, and the propeller torques are constrained to be positive to eliminate the possibility of negative power draws for windmilling. For the cruise propeller, the torque is given a minimum of $50 \mathrm{Nm}$ to stay within the range of optimal motor efficiencies since the optimizer may try to go lower during climb and descent. The tip-over constraint enforces a minimum ground-clearance angle of $20^{\circ}$ and the high-lift propeller tip speed corresponds to a maximum of $450 \mathrm{ft} / \mathrm{s}$, added for noise considerations. The failure constraint ensures the Von Mises stress is below yield with a 1.5 factor of safety and a 2.5 multiplier for a pull-up maneuver.

\section{B. Multi-point MDO: a cruise condition and a high-lift condition}

\section{Problem description}

In this problem, we perform MDO with one on-design and one off-design point. The on-design point is the cruise condition as described in Tab. 1, and the off-design point is the high-lift sizing condition at sea level with a $10 \%$ lift margin.

The objective function is range, which is given by

$$
R=W_{b a t t}(E / W)_{b a t t} v / P_{r e q},
$$

where $W_{\text {batt }}$ is the weight of the battery, $(E / W)_{b a t t}$ is the energy density of the battery, $v$ is the airspeed, and $P_{r e q}$ is the power required. We make the approximation that the power required is constant throughout the flight and is equal to the cruise value. Energy consumption has been used previously as the objective function for an optimization study involving the full mission profile [8], but here we maximize range, allowing the battery sizing to vary in the design problem.

\section{Results}

As was discussed previously, we solve four MDO problems that build up to the full problem, which are listed in Tab. 4. The total optimization time varies significantly depending on the case, but the mean is roughly an hour using a 2.8 $\mathrm{GHz}$ Intel Xeon E5-2680v2 processor. This corresponds to a few hundred model evaluations. The run times do not show a significant correlation with the size of the problem when comparing case MP0, MP1, MP2, and MP3.

In Tab. 5, we find that optimizing the battery weight alone (MP1) results in a $4.5 \%$ increase in range, and optimizing the cruise propeller diameter (MP2) produces a $10.7 \%$ increase in range compared to the baseline optimization result. The MP2 result decreases the diameter to roughly $4.35 \mathrm{ft}$, although not to the lower bound of $4 \mathrm{ft}$. Decreasing the cruise propeller diameter allows the wing span and high-lift propeller diameters to increase while satisfying the tip-over constraint. Evidently, the algorithm finds it worthwhile to sacrifice cruise propeller efficiency and accept an increase in structural weight for better aerodynamic efficiency through a larger wing span and better high-lift performance through larger high-lift propellers. Adding the wing twist distribution design variables (MP3) results in an additional $5.4 \%$ range improvement with negligible change in cruise propeller diameter compared to MP2. However, the validity of the MP3 result requires further investigation because optimizing the twist distribution exposes the details of the 
propeller-wing aerodynamic model, especially the wing stall model. In all cases, the range increase is achieved primarily through an increase in battery weight, which is enabled by an increase in wing span.

\begin{tabular}{lllllll}
\hline Problem & $\begin{array}{l}\text { Battery } \\
\text { weight }(\mathrm{lb})\end{array}$ & $\begin{array}{l}\text { Cruise } \\
\text { diam. }(\mathrm{ft})\end{array}$ & $\begin{array}{l}\text { High-lift } \\
\text { diam. }(\mathrm{ft})\end{array}$ & $\begin{array}{l}\text { Wing span } \\
(\mathrm{ft})\end{array}$ & $\begin{array}{l}\text { Range } \\
(\mathrm{nmi})\end{array}$ & $\begin{array}{l}\text { Increase } \\
\text { in range }\end{array}$ \\
\hline MP0 & 1346 & 5.00 & 1.76 & 30.12 & 177.1 & \\
MP1 & 1441 & 5.00 & 1.84 & 31.08 & 185.1 & $4.5 \%$ \\
MP2 & 1607 & 4.35 & 2.04 & 32.86 & 196.0 & $10.7 \%$ \\
MP3 & 1701 & 4.30 & 2.06 & 32.98 & 205.7 & $16.1 \%$ \\
\hline
\end{tabular}

Table 5: Optimization results for the cruise MDO with high-lift constraints. Values in red are fixed during optimization.

\section{Full-mission MDO \\ 1. Problem description}

In this problem, we extend the previous MDO problem by considering the full mission profile, discretized with 10 points. As shown in Tab. 4, we now include the parametrized altitude and velocity profiles as design variables, and enforce the horizontal and vertical equations of motion at all points. The initial and final altitude values are constrained to be zero, and the initial and final velocities are constrained to be 58 knots and 75 knots, respectively for climb and approach. The altitude profile is also constrained to be below 8000 feet at all points due to cabin pressurization considerations.

Most of the design variables are similar to those in the multi-point MDO problem. Cruise and high-lift propeller rotational speed, angle of attack, and cruise propeller pitch are separate design variables at each mission point. Here, a new design variable is added-a high-lift switch variable. When this variable is 0 , the Fowler flaps are retracted and the high-lift propellers are considered folded in and the high-lift torque, thrust, and power are zero; when this variable is 1, the flaps are deployed and the high-lift propellers are assumed to be operational. A value strictly between 0 and 1 represents a partial deployment of flaps and a fractional multiplier for the high-lift torque, thrust, and power-this formulation contributes error when the optimal solution has values that are not at 0 or 1 . However, this gives the optimizer full control of the deployment of the flaps and high-lift propellers at each point in the mission.

Here, range is a design variable subject to a constraint. The constraint is that the total energy available from the battery is equal to the energy used over the mission. The energy available is simply the product of the energy density and battery weight. The constraint is then

$$
W_{\text {batt }}(E / W)_{\text {batt }}=\int_{\text {mission }} P_{\text {req }} d t
$$

which we can reformulate as

$$
W_{\text {batt }}(E / W)_{\text {batt }}=\int_{\text {mission }} \frac{P_{r e q}}{v_{x}} d x,
$$

where $v_{x}$ is the horizontal velocity component. We can discretize and approximate in terms of the known velocity $v$ and altitude $h$ profiles:

$$
W_{\text {batt }}(E / W)_{b a t t}=\sum^{n}\left[\frac{P_{r e q}}{v}\left(\sqrt{\frac{d h^{2}}{d x}+1}\right) \Delta x\right],
$$

where $n$ is the number of mission points, and $x$ is the horizontal coordinate. In the full-mission formulation, we also impose a constraint on maximum power draw to ensure this value is feasible given the power density of the battery.

\section{Results}

As we did for the multi-point MDO formulation, we solve four MDO problems: a baseline optimization, the same problem with battery weight added, the second problem with cruise propeller diameter added, and then the full problem including wing twist distribution. With 10 mission points, the computation times for the full-mission MDO have a mean of 10 hours on the same hardware as the MP cases. The results are shown in Tab. 6. 


\begin{tabular}{lllllll}
\hline Problem & $\begin{array}{l}\text { Battery } \\
\text { weight }(\mathrm{lb})\end{array}$ & $\begin{array}{l}\text { Cruise } \\
\text { diam. }(\mathrm{ft})\end{array}$ & $\begin{array}{l}\text { High-lift } \\
\text { diam. }(\mathrm{ft})\end{array}$ & $\begin{array}{l}\text { Wing span } \\
(\mathrm{ft})\end{array}$ & $\begin{array}{l}\text { Range } \\
(\mathrm{nmi})\end{array}$ & Change \\
\hline FM0 & 1346 & 5.00 & 1.84 & 31.08 & 118.7 & \\
FM1 & 1235 & 5.00 & 1.84 & 31.08 & 120.3 & $1.4 \%$ \\
FM2 & 1440 & 4.05 & 2.13 & 33.67 & 133.1 & $12.1 \%$ \\
FM3 & 1571 & 4.00 & 2.15 & 33.81 & 140.3 & $18.2 \%$ \\
\hline
\end{tabular}

Table 6: Optimization results for the MDO including the full mission profile. Values in red are fixed during optimization.

The range predictions are much more conservative than in the cruise MDO problem, since here, the range is more accurately computed using the power and velocity profiles over the full mission, rather than a single representative cruise point. The performance and energy usage at the high-lift conditions are inherently included in this range estimate.

We observe a $12.1 \%$ increase in the cruise propeller optimization (FM2) compared to the baseline (FM0), which is similar to the $10.7 \%$ increase we saw with MP2 compared to MP0. Interestingly, FM2 achieves the $12.1 \%$ increase through only a $7.0 \%$ larger battery, while MP2 achieves the $10.7 \%$ increase with a $19.4 \%$ larger battery. Again, we see that the optimizer prefers a lower cruise propeller diameter although in this case, the optimized value is nearly at the lower bound of $4 \mathrm{ft}$. The optimization with twist included (FM3) adds a $6.1 \%$ improvement in addition to the $12.1 \%$ improvement in FM2, which is in line with the 5.4\% that we saw earlier, comparing MP3 to MP2.

As we saw in the MP cases, the range improvements are primarily obtained through a larger battery, which is in turn enabled by a larger wing span that supplies the extra lift. Figure 5 illustrates why the cruise propeller diameter must be decreased to allow the wing span and high-lift propeller diameter to increase while satisfying the tip-over constraint. The front view of the aircraft is shown for the FM0 and FM2 optima, which represent the baseline design and the design after optimizing all the variables except for the twist distribution.

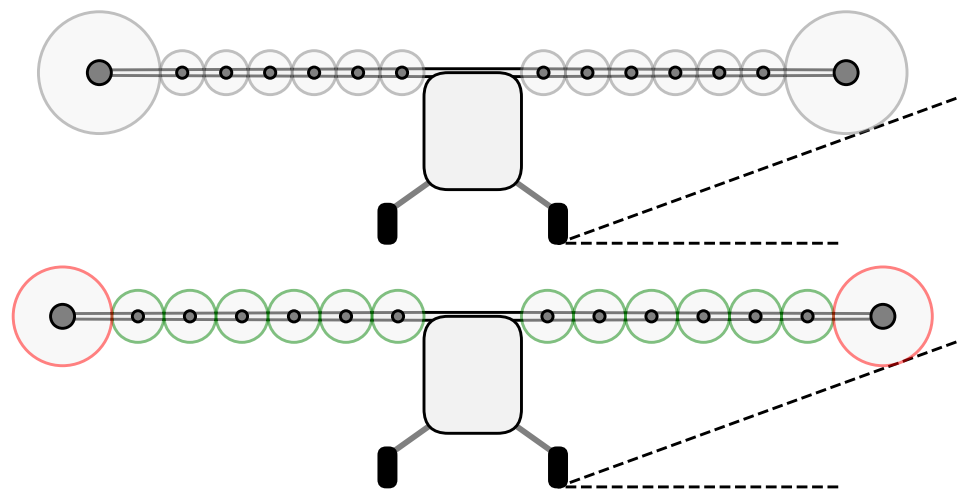

Figure 5: The front view of the aircraft for the baseline (FM0, top) and optimized (FM2, bottom) configurations. Because of the tip-over constraint, a decrease in cruise propeller (red) diameter is required to allow the high-lift (green) propeller and wing span to increase.

Overall, our conclusion in comparing the full-mission results to the multi-point results is that while the absolute range values differ significantly (on the order of 50\%), the qualitative observations match between the FM and MP results. These are: the cruise propeller diameter decreases, the wing span and high-lift propeller diameter increase, optimizing the battery weight and cruise propeller diameter results in a $\mathcal{O}(10 \%)$ range increase, and the range improvements are obtained by carrying a larger battery which in turn is enabled by a larger wing span.

Figures 6, 7 and 8 plot various aspects of the optimized configuration for the full-mission MDO problems. In Fig. 6, we see that the altitude profiles go to the cabin pressurization limit of $8000 \mathrm{ft}$. The optimized profiles for the four cases look very similar for this reason, as the optimal design tries to fly as high as possible for most of cruise. The velocity profiles agree with intuition as well, as the aircraft speeds up to just over 150 knots. We note that the minimum average velocity constraint of roughly 135 knots is active in all four cases and is chosen somewhat arbitrarily. Changing this lower bound is expected to change the design; this is one of the reasons why we perform a baseline optimization and 
emphasize the percent increase in range rather than the absolute range value itself. The high-lift propellers' rotational speeds are limited by the tip-speed constraint of $450 \mathrm{ft} / \mathrm{s}$ as we see in Fig. 6.
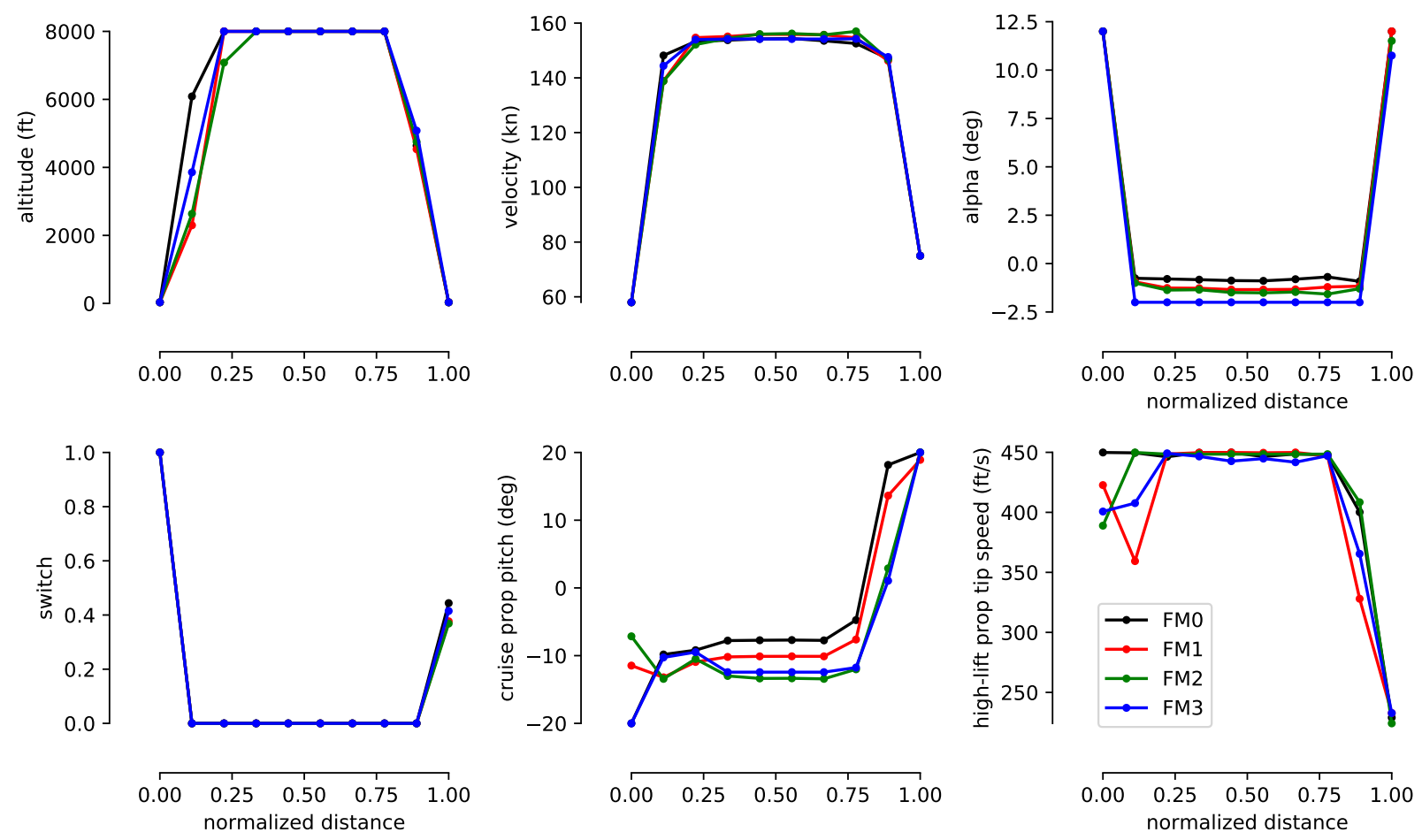

Figure 6: Optimized mission profiles versus normalized distance for the full-mission MDO.

We see in Fig. 6 that the switch variable is 1 at takeoff, when the flaps and high-lift propellers are the most needed, and remain zero for most of cruise to save energy. Near the end of the mission, they go to a partially deployed state. An unexpected result is that the high-lift propellers are unused during descent, as we see from the high-lift propeller power plots. One would expect full flap deployment and use of the high-lift propellers to enable a slow approach speed. However, they are not used because the aircraft glides with a high descent angle so that the drag helps counteract the weight. We expect that we would see full high-lift propeller operation in future work if we assume a step descent profile or if we use a higher-resolution discretization of the mission profile.

In Fig. 7, we can see the details of the propeller operation throughout the mission. The cruise propellers speed up to the maximum of 3000 RPM in FM2 and FM3 since the diameters are smaller. We see the opposite for the high-lift motors; the rotational speed is lower in FM2 and FM3 because the high-lift propeller diameters are smaller. In all four optimization problems, the tip speed is the driving constraint for the high-lift propeller RPM. We see that maximum the cruise propeller power is roughly $60 \mathrm{~kW}$ in all four cases, which is close to the $65 \mathrm{~kW}$ nominal power on the X-57. The maximum power draw for the high-lift propellers is just under $10 \mathrm{~kW}$, which is also close to nominal values.

In Fig. 8, we see that the propellers optimize to monotonically decreasing chord profiles. This is expected for the high-lift propellers because such a blade shape is efficient for lift augmentation, but this is unexpected for the cruise propellers and merits further investigation in future work. The cruise propellers are utilized more during cruise, where efficient thrust generation is the goal, rather than lift augmentation. As expected, the FM2 and FM3 high-lift blades are thinner since the overall diameter is larger.

Examining Fig. 8, we see the consequence of decreasing the cruise propeller diameter. FM2 and FM3, which have the smaller cruise propellers, consume more power during the cruise portions of the mission where only the cruise propellers are active. Therefore, they sacrifice thrust-generation efficiency in the cruise propellers for the sake of a larger wing. 

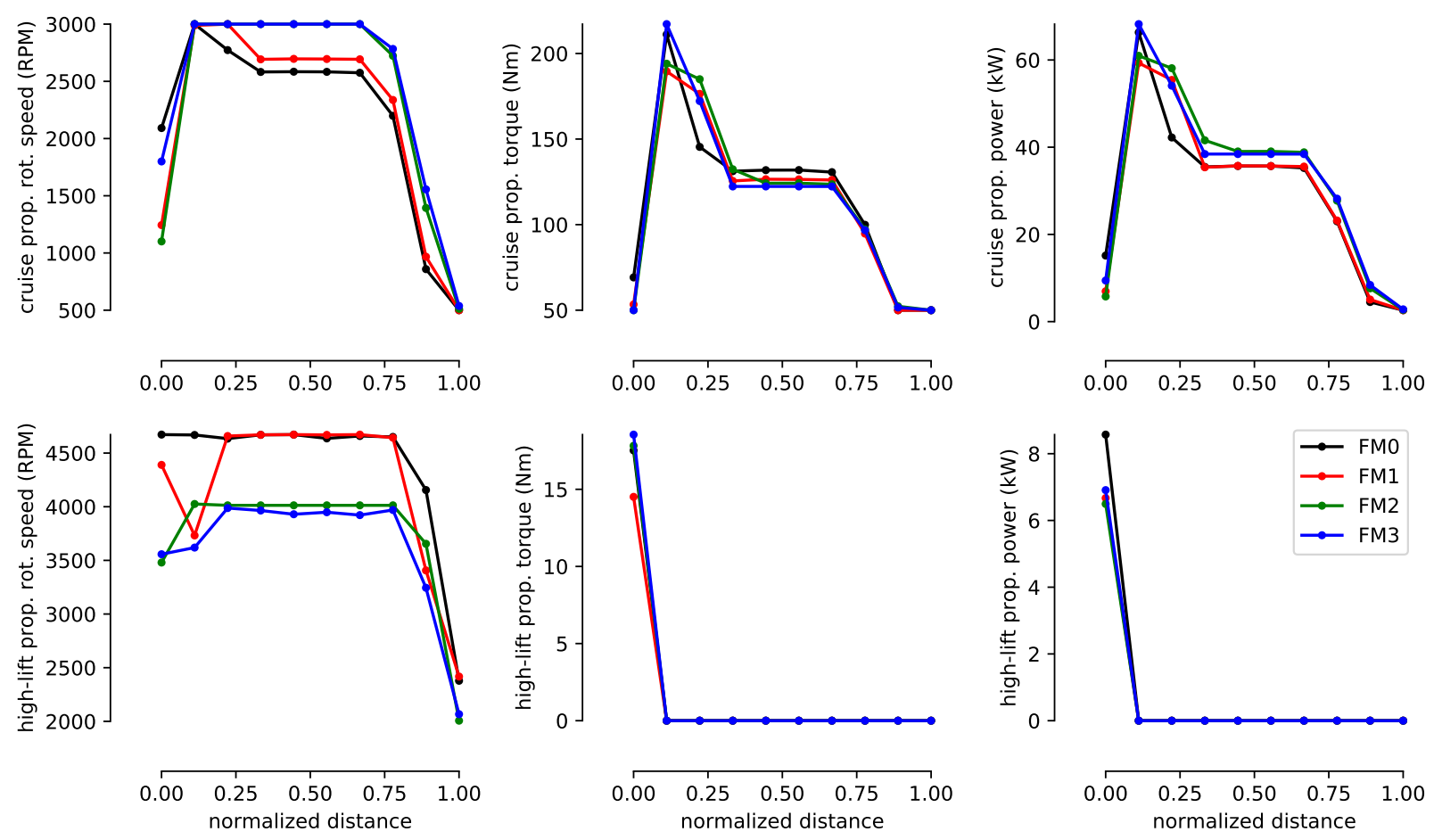

Figure 7: Optimized propeller-related profiles versus normalized distance for the full-mission MDO.
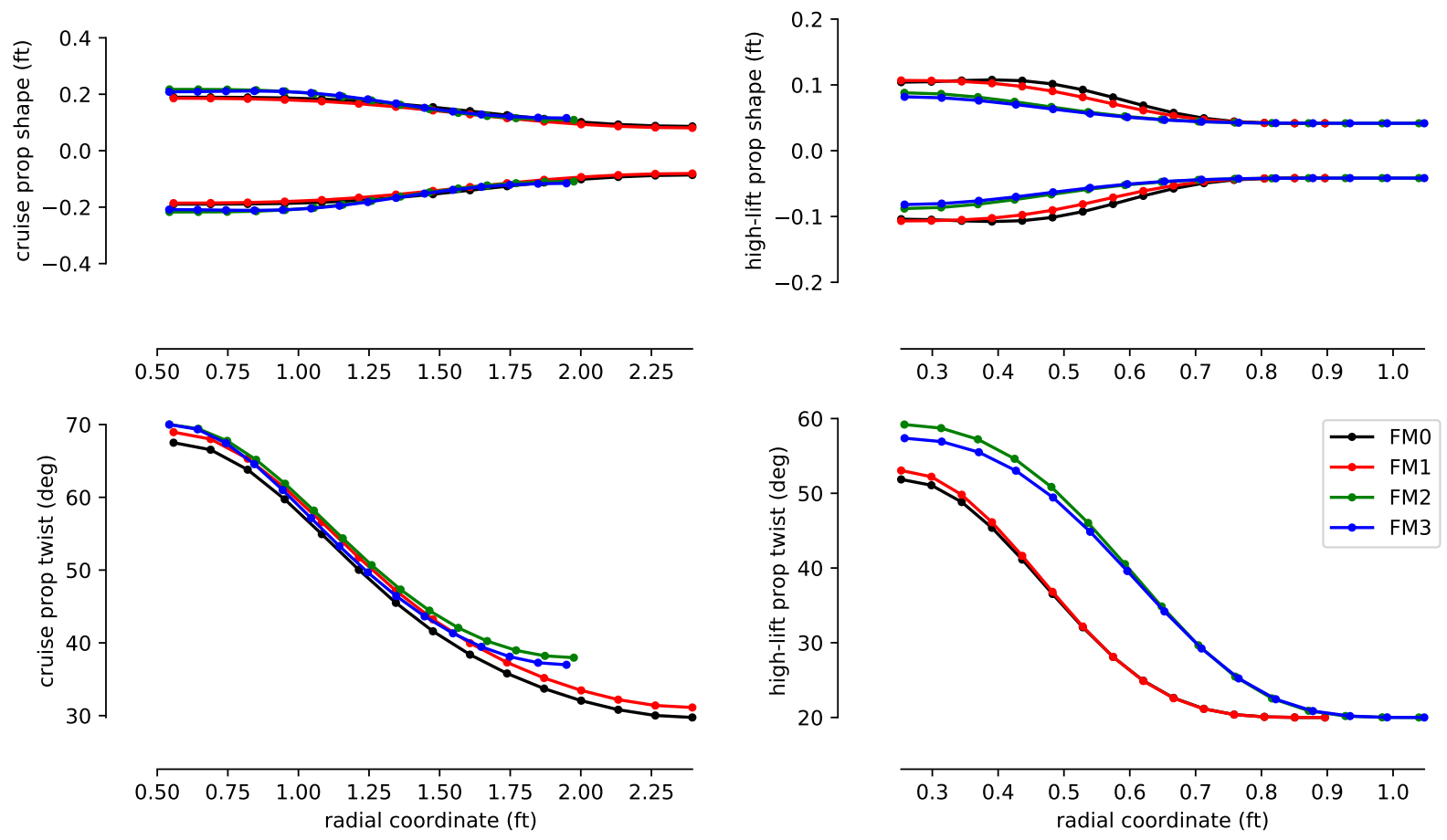

Figure 8: Optimized propeller designs for the full-mission MDO. 


\section{Conclusion}

In this paper, we apply large-scale multidisciplinary optimization methods to an electric aircraft for on-demand mobility, NASA's X-57 Maxwell X-plane. Our main objective is to explore whether multidisciplinary design optimization (MDO) can be an effective tool in the design of an electric aircraft with distributed electric propulsion (DEP). We summarize the results of this paper with three conclusions.

First, we find that for the X-57, MDO results in a 12\% increase in range-the objective function. This improvement is primarily obtained by decreasing the cruise propeller diameter, which enables increases in the high-lift propeller diameter and the wing span. This result implies that the performance metric of interest—in this case, range-benefits more from the aerodynamic efficiency increase due to the higher aspect ratio wing than it does from more efficient thrust generation from the cruise propellers. Further model validation and investigation are necessary to confirm the findings, but these results suggest that MDO can make non-trivial changes to the performance of electric aircraft designs using non-intuitive design changes that exploit tradeoffs.

Second, we demonstrate that a modular adjoint approach to the construction of the multidisciplinary model is worthwhile, as it enables efficient solution of an MDO problem incorporating mission, propulsion, aerodynamics, and structures. The largest MDO problem we consider contains 122 design variables and 74 constraints, and the optimization problem is solved at the cost of only $\mathcal{O}(100)$ evaluations of the model. The adjoint-based approach affords us the luxury of adding disciplines and design variables at will during iterative testing and development of the model. For instance, including the wing twist distribution in the MDO problem, which represents 21 additional design variables, produced no noticeable increase in computation time.

Third, we find that there are large differences in the predicted performance and the optimal designs, when performing MDO at a representative cruise condition versus MDO considering the full mission profile. Due to the short ranges of electric aircraft concepts, the optimal designs and range predictions are vastly different when simplifying by considering representative cruise conditions subject to off-design constraints, as is commonly done in commercial aircraft design. Although we find full-mission MDO to be an order of magnitude more expensive, it is necessary in this context.

As we state in the discussion of the models, there is significant room for improvement in the multidisciplinary model. We identify three areas in particular for future work. The first area is improving the wing aerodynamics model by integrating 2-D airfoil section data in the vortex lattice method analysis. This would also capture stall more accurately, rather than simply imposing a maximum section lift coefficient. The second area is running with more mission discretization points. Currently, the full-mission MDO has a runtime in the neighborhood of 10 hours, depending on the case. With a larger computational budget, it would be beneficial to run with more points to smooth out some spurious effects due to the coarseness of the discretization and to obtain more accurate range predictions. The third area is further validation and refinement of the propeller-wing interaction model, as this would impact the propeller design and sizing. An improved and validated model would allow us to investigate the finding that reducing the cruise propeller diameters is beneficial. On a longer-term scale, another avenue for future work is applying similar methods in the design of tilt-wing electric aircraft for urban air mobility.

\section{Acknowledgments}

The first author was supported by the NASA ARMD Transformational Tools and Technologies Project. The authors would like to thank Dr. Nicholas K. Borer for insightful discussions and valuable feedback. 


\section{References}

[1] Moore, M. D., “The third wave of aeronautics: On-demand mobility,” Tech. rep., SAE Technical Paper, 2006.

[2] Holmes, B. J., "A Vision and Opportunity for Transformation of On-Demand Air Mobility," 16th AIAA Aviation Technology, Integration, and Operations Conference, 2016, p. 3465.

[3] Antcliff, K. R., Moore, M. D., and Goodrich, K. H., "Silicon Valley as an Early Adopter for On-Demand Civil VTOL Operations," 16th AIAA Aviation Technology, Integration, and Operations Conference, 2016, p. 3466.

[4] Vascik, P. D. and Hansman, R. J., "Evaluation of Key Operational Constraints Affecting On Demand Mobility for Aviation in the Los Angeles Basin: Ground Infrastructure, Air Traffic Control and Noise," 17th AIAA Aviation Technology, Integration, and Operations Conference, 2017.

[5] Borer, N. K., Nickol, C. L., Jones, F., Yasky, R., Woodham, K., Fell, J., Litherland, B., Loyselle, P., Provenza, A., Kohlman, L., et al., "Overcoming the Adoption Barrier to Electric Flight," 54th AIAA Aerospace Sciences Meeting, 2016, p. 1022.

[6] Vascik, P. D. and Hansman, R. J., "Constraint Identification in On Demand Mobility for Aviation through an Exploratory Case Study of Los Angeles," 17th AIAA Aviation Technology, Integration, and Operations Conference, 2017.

[7] Stoll, A. M. and Veble Mikic, G., "Design Studies of Thin-Haul Commuter Aircraft with Distributed Electric Propulsion," 16th AIAA Aviation Technology, Integration, and Operations Conference, 2016, p. 3765.

[8] Falck, R. D., Chin, J. C., Schnulo, S. L., Burt, J. M., and Gray, J. S., “Trajectory Optimization of Electric Aircraft Subject to Subsystem Thermal Constraints," 18th AIAA/ISSMO Multidisciplinary Analysis and Optimization Conference, 2017 , p. 4002.

[9] Borer, N. K., Patterson, M. D., Viken, J. K., Moore, M. D., Bevirt, J., Stoll, A. M., and Gibson, A. R., "Design and Performance of the NASA SCEPTOR Distributed Electric Propulsion Flight Demonstrator," 16th AIAA Aviation Technology, Integration, and Operations Conference, 2016, p. 3920.

[10] Borer, N. K., Moore, M. D., and Turnbull, A., "Tradespace Exploration of Distributed Propulsors for Advanced On-Demand Mobility Concepts," 14th AIAA Aviation Technology, Integration, and Operations Conference, 2014, p. 2850.

[11] Borer, N. K. and Moore, M. D., "Integrated Propeller-Wing Design Exploration for Distributed Propulsion Concepts," 53rd AIAA Aerospace Sciences Meeting, 2015, p. 1672.

[12] Patterson, M. D., Borer, N. K., and German, B. J., "A Simple Method for High-Lift Propeller Conceptual Design," 54th AIAA Aerospace Sciences Meeting, 2016, p. 0770.

[13] Bramesfeld, G. and Maughmer, M. D., "Relaxed-wake vortex-lattice method using distributed vorticity elements," Journal of aircraft, Vol. 45, No. 2, 2008, pp. 560-568.

[14] Patterson, M. D., Daskilewicz, M. J., and German, B. J., "Conceptual design of electric aircraft with distributed propellers: multidisciplinary analysis needs and aerodynamic modeling development," Proceedings of the 52nd AIAA Aerospace Sciences Meeting, 2014, p. 0534.

[15] Cole, J. A., Maughmer, M. D., Bramesfeld, G., and Kinzel, M., "A Higher-Order Free-Wake Method for Propeller-Wing Systems," 35th AIAA Applied Aerodynamics Conference, 2017, p. 3414.

[16] Stoll, A. M., Bevirt, J., Moore, M. D., Fredericks, W. J., and Borer, N. K., "Drag reduction through distributed electric propulsion," 14th AIAA Aviation Technology, Integration, and Operations Conference, 2014, p. 2851.

[17] Kao, J. Y., Hwang, J. T., Martins, J. R. R. A., Gray, J. S., and Moore, K. T., "A modular adjoint approach to aircraft mission analysis and optimization," 56th AIAA/ASCE/AHS/ASC Structures, Structural Dynamics, and Materials Conference, Jan 2015. doi:10.2514/6.2015-0136.

[18] Hwang, J. T. and Martins, J. R. R. A., "Allocation-mission-design optimization of next-generation aircraft using a parallel computational framework," 57th AIAA/ASCE/AHS/ASC Structures, Structural Dynamics, and Materials Conference, San Diego, CA, Jan 2016.

[19] Sobieszczanski-Sobieski, J., "Sensitivity of complex, internally coupled systems," AIAA journal, Vol. 28, No. 1, 1990, pp. 153-160.

[20] Martins, J. R. R. A. and Hwang, J. T., "Review and Unification of Methods for Computing Derivatives of Multidisciplinary Computational Models," AIAA Journal, Vol. 51, No. 11, November 2013, pp. 2582-2599. doi:10.2514/1.J052184.

[21] Hwang, J. T., A modular approach to large-scale design optimization of aerospace systems, Ph.D. thesis, University of Michigan, 2015.

[22] Gray, J., Moore, K. T., and Naylor, B. A., "OpenMDAO: An Open Source Framework for Multidisciplinary Analysis and Optimization," Proceedings of the 13th AIAA/ISSMO Multidisciplinary Analysis Optimization Conference, Fort Worth, TX, Sept. 2010, AIAA 2010-9101.

[23] Ning, S. A., "A simple solution method for the blade element momentum equations with guaranteed convergence," Wind Energy, Vol. 17, No. 9, 2014, pp. 1327-1345.

[24] Hwang, J. T. and Martins, J. R. R. A., “A fast-prediction surrogate model for large datasets," Aerospace Science and Technology, 2017. 
[25] Veldhuis, L. L. M., Propeller Wing Aerodynamic Interference, Ph.D. thesis, Delft University of Technology, 2005.

[26] Conway, J. T., “Analytical solutions for the actuator disk with variable radial distribution of load," Journal of Fluid Mechanics, Vol. 297, 1995, pp. 327-355.

[27] Alba, C., Elham, A., Veldhuis, L. L., and German, B. J., "A Surrogate-Based Multi-Disciplinary Design Optimization Framework Exploiting Wing-Propeller Interaction," 18th AIAA/ISSMO Multidisciplinary Analysis and Optimization Conference, 2017, p. 4329.

[28] Jasa, J. P., Hwang, J. T., and Martins, J. R. R. A., “Open-source coupled aerostructural optimization using Python,” Structural and Multidisciplinary Optimization, 2018, (Accepted subject to revisions on October 20, 2017).

[29] Borer, N. K., Derlaga, J. M., Deere, K. A., Carter, M. B., Viken, S. A., Patterson, M. D., Litherland, B. L., and Stoll, A., "Comparison of Aero-Propulsive Performance Predictions for Distributed Propulsion Configurations," 55th AIAA Aerospace Sciences Meeting, 2017, p. 0209.

[30] Deere, K. A., Viken, S. A., Carter, M. B., Viken, J. K., Derlaga, J. M., and Stoll, A. M., "Comparison of High-Fidelity Computational Tools for Wing Design of a Distributed Electric Propulsion Aircraft," Aviation Forum, AIAA, Denver, Colorado, 2017, pp. 5-9.

[31] Kreisselmeier, G. and Steinhauser, R., "Systematic Control Design by Optimizing a Vector Performance Index," International Federation of Active Controls Syposium on Computer-Aided Design of Control Systems, Zurich, Switzerland, 1979. 